\title{
Introduction to a New Review Section
}

This journal has occasionally published reviews of selected topics in the pharmacological sciences. These reviews, however, have been few and far between. Now we perceive the need to publish more frequent reviews of important topics in the pharmacological sciences by authorities in their respective fields. They will place their own work in a broad context. The intent is to provide readers an opportunity to extend their interests to important fields beyond their own immediate pharmacological research. We believe that such reviews, initiated by Spector's accompanying authoritative overview of drug transport in the central nervous system, should enhance the value of this journal.

The increasing complexity of biological and chemical science is magnified in pharmacological science due to its integrative nature. With the vast expansion of data from different fields, often fragmented, of varying degrees of quality, and published in a wide variety of journals and other media, there is an urgent need for an integrative, systematic approach to complex pharmacological subjects. This approach could be termed Pharmacological Hermeneutics. What criteria are required for valid statements, conclusions? What are the data? Since there are multiple sources and types of data, one needs to be sure the data are 'consistent' with the conclusions drawn.

The accompanying review entitled 'Drug Transport in the Mammalian Central Nervous System: Multiple Complex Systems. A Critical Analysis and Commentary' attempts an integrative, systematic approach to Pharmacokinetics of the CNS. It provides for the pharmacology community the general principles, nature of proof, types of required experiments, and a balanced interpretation of this field illustrated with 5 interesting, important, and practical examples. This approach offers a general model which hopefully will stimulate other reviews of various timely topics.

Elliot S. Vesell, MD, ScD, Editor

Melvin L. Billingsley, $\mathrm{PhD}$, Associate Editor 